\title{
Oral Health Behaviors in Very Young Children in Low-Income Urban Areas in Chicago, Illinois, 2018-2019
}

\author{
Molly Martin, MD, MAPP ${ }^{1,2}$; Oksana Pugach, $\mathrm{PhD}^{2}$; David Avenetti, DDS, MPH ${ }^{2,3}$; Helen Lee, MD, MPH ${ }^{2,4}$; \\ Shojanny Salazar, $\mathrm{MD}^{2}$; Genesis Rosales, $\mathrm{MSW}^{2}$; Nattanit Songthangtham, $\mathrm{MPH}^{2}$
}

\begin{abstract}
Accessible Version: www.cdc.gov/pcd/issues/2020/20_0213.htm
Suggested citation for this article: Martin M, Pugach O, Avenetti D, Lee H, Salazar S, Rosales G, et al. Oral Health Behaviors in Very Young Children in Low-Income Urban Areas in Chicago, Illinois, 2018-2019. Prev Chronic Dis 2020; 17:200213. DOI: https://doi.org/10.5888/pcd17.200213.
\end{abstract}

\section{PEER REVIEWED}

\section{Summary}

What is already known on this topic?

Health disparities are well documented in the prevalence of and morbidity associated with dental caries, the most common chronic disease of childhood.

What is added by this report?

Most data on oral health risk and behaviors do not include infants and toddlers. We describe the oral health behaviors of children younger than 3 years and identify areas for intervention.

What are the implications for public health practice?

Behaviors established during early childhood set the trajectory for a lifetime. This analysis shows the importance of the family unit and social support in efforts to improve oral health outcomes for high-risk children.

\section{Abstract}

\section{Introduction}

Because most data on oral health do not include infants and toddlers, we aimed to describe the oral health behaviors of lowincome children younger than 3 years and determine factors associated with child tooth brushing.

\section{Methods}

We obtained data from the Coordinated Oral Health Promotion Chicago study, which included 420 families with children aged 6 to 36 months and their caregivers in Cook County, Illinois. We as- sessed child frequency of brushing from caregiver reports and objectively determined child dental plaque scores. Significant factors associated with tooth brushing frequency and dental plaque score were identified using the Least Absolute Shrinkage and Selection Operator variable selection.

\section{Results}

Mean child age was 21.5 (SD, 6.9) months, and only $45 \%$ of caregivers brushed their children's teeth twice per day or more. The mean plaque score was 1.9 (SD, 0.6), indicating high levels of plaque. Child brushing frequency was higher when children were older; used the correct toothpaste amount; brushed for a longer duration; and when caregivers brushed their own teeth more frequently, had more help with the overall care of the child's teeth, and had family to help. Child brushing frequency was lower for caregivers with more interference from activities of daily life. Children whose caregivers had more adult help with child brushing had better plaque scores; worse plaque scores were seen in children with higher sugary beverage and food consumption and lower household incomes.

\section{Conclusion}

The tooth brushing behaviors of young children are strongly associated with those of their parents and with the level of family support for brushing. Interventions to improve brushing in young children should focus on the entire family.

\section{Introduction}

Dental caries is the most common chronic disease of childhood, affecting over half of US children aged 6 to 8 years (1). Although treatment of caries leads to significant direct health care costs, the true costs extend beyond the health care setting. Caries is associated with impaired cognitive development, increased school absenteeism, worse school performance, increased missed work for parents, and worse quality of life (2-4). Oral health disparities are 
well documented, and low-income minority children experience the highest prevalence and illness from caries (5-8).

Caries risk is influenced by many factors over the life course beginning during the prenatal period (9). Most data on oral health risk and behaviors do not include infants and toddlers, even though these formative years determine the trajectory for children's oral health (10). The largest health survey in the United States, the National Health and Nutritional Examination Survey (NHANES), captures oral health behaviors data only for children aged 3 years or older. Also lacking is a complete understanding of oral health risk factors in very young children and a reliable model to predict future caries in children. Although many of these risk factors are well documented (eg, low fluoride exposure, limited access to dental care, overconsumption of sugar-sweetened beverages) $(11,12)$, the frequency of exposure to these risk factors in children younger than 3 years is unknown. This lack of data makes it challenging to prospectively identify children who will develop caries and become high users of tertiary oral health services such as emergency departments, urgent care clinics, and operating rooms for oral care.

In addition to limited information on oral health risk factors for children younger than 3 , information on the frequency and facilitators of protective oral health behaviors in this age group is also lacking. Major health promotion efforts have been implemented to educate primary care providers and families about these protective behaviors $(13,14)$. One of the primary recommendations is twice-daily tooth brushing with fluoridated toothpaste, shown to be a low-cost clinically effective means of reducing caries for dentate children (15). Chronic conditions such as childhood obesity and diabetes have resulted in an increased awareness of the need to reduce sugar-sweetened beverages and high-sugar foods. There is a growing emphasis on the age 1 dental visit and increased coverage for private and publicly funded dental programs (16-18). Although access to dental care theoretically has improved with expanded programs and Medicaid coverage, many barriers to accessing care persist because dental coverage does not equate to use of dental care (7). Whether increased awareness of brushing and dietary recommendations translates to more adoption of these behaviors in young children is also unknown.

To effectively implement preventive interventions to establish healthy oral care behaviors, we must first characterize the baseline oral health behaviors of young children and identify factors associated with these behaviors. The Coordinated Oral Health Promotion (CO-OP) Chicago study included 420 children aged 6 to 36 months and their primary caregivers. In this analysis we describe the children's oral health behaviors and determine factors associated with child tooth brushing, captured as caregiver-reported brushing frequency, and observed dental plaque.

\section{Methods}

Data were obtained from the baseline sample $(\mathrm{N}=420$ child/caregiver dyads) of the CO-OP Chicago study with the National Institute of Dental and Craniofacial Research's Oral Health Disparities Consortium (19). To qualify, families needed to have a child aged 36 months or younger with at least 2 fully erupted central maxillary incisors. Children also had to receive medical care or services at one of the partnering Special Supplemental Nutrition Program for Women, Infants, and Children (WIC) centers or pediatric medical clinics serving low-income communities in Cook County, Illinois. Families were excluded if their primary language was not English or Spanish, the child did not live with the primary caregiver 5 days per week or more, or the child had medical conditions that interfered with routine tooth brushing. Participants were recruited by research assistants (RAs) in the 20 partnering clinics and WIC centers from January 2018 through February 2019. Families that met inclusion criteria were scheduled for an enrollment visit where the baseline data collection occurred (19).

Caregivers provided written informed consent and parental permission at the start of the enrollment visit. Child assent was waived because of child age. Institutional review boards at the University of Illinois at Chicago, the University of California San Francisco, and the Chicago Department of Public Health approved the study.

Data collection was conducted mainly in homes by paired RAs using standardized methods established by the research team (19). RAs first administered a verbal questionnaire using prompt cards that asked about the child's and caregiver's oral health behaviors and beliefs, other health conditions, access to care, psychosocial factors, and demographics. Child and caregiver oral health quality of life was captured using the Early Childhood Oral Health Impact Scale (ECOHIS) and Oral Health Impact Profile, respectively. Caregiver quality of life (referred to as "social functioning"), depression, anxiety, and social support were measured using PatientReported Outcomes Measurement Information System (PROMIS) measures. Family functioning was assessed using the Confusion, Hubbub, and Order Scale (CHAOS). RAs took photographs of the child's teeth before and after the application of plaque disclosing solution using a standardized protocol. At the end of the visit, caregivers were asked to demonstrate how the child's teeth are typically brushed. RAs used checklists to systematically capture duration of brushing, supplies used (eg, toothbrushes, mouthwash, floss), and parent involvement. Data were entered directly into the study's Research Electronic Data Capture (REDCap) database. Calibrated dental clinicians, including a board-certified pediatric dentist and a registered dental hygienist, later reviewed images in the research office and scored them for plaque using the Oral Hy-

The opinions expressed by authors contributing to this journal do not necessarily reflect the opinions of the U.S. Department of Health and Human Services, the Public Health Service, the Centers for Disease Control and Prevention, or the authors' affiliated institutions. 
giene Index-Maxillary Incisor Simplified (OHI-MIS) scale. The OHI-MIS scale is a modification of the Simplified Oral Hygiene Index; the adaptions allow for plaque scoring using photographs in children with an incomplete primary dentition (20). Plaque scores of less than 0.7 are considered "good," $0.7-1.8$ are "fair," and $1.9-3.0$ are "poor" (21).

\section{Analyses}

Demographic characteristics of children and caregivers, as well as the frequency of tooth brushing and plaque scores, were reported using counts (percentages) or mean and SD for categorical variables and median and interquartile range for continuous variables. Frequency of brushing was recoded as a continuous variable, and variables with 5 or more ordinal categories were also recoded as continuous measures. Thirty-two variables were considered in the analyses based on a priori-determined potential for influence on the primary outcomes. Pair-wise correlations for most covariates were low, with few correlations being in the moderate range, specifically among the PROMIS measures $(\rho<0.80)$. The 2 primary outcome measures, child frequency of brushing and plaque score, were correlated at $-0.11, P=.02$. Some variables had missing values. Of 420 participant records, 369 (87.9\%) had complete observational data. Variables describing observed toothpaste amount, type of toothpaste, and length of observed brushing had the most missing data points ( $47-48$ cases, $11.2 \%-11.4 \%$ ) due mainly to parent or child refusal of the brushing demonstration. Responses were coded as "not applicable" for some variables. For example, if a child had not yet started brushing, toothpaste use would be coded as not applicable. Those responses were then recoded as no for analysis. Household income was reported as unknown by $52 \%$ of participants, which was expected for the population. Consequently, income was used as a categorical variable, with "unknown" as a category.

The selection of significant factors associated with the 2 outcomes (frequency of tooth brushing and plaque score) was performed using the Least Absolute Shrinkage and Selection Operator (LASSO) variable selection. The LASSO is a shrinkage estimator with a variable selection. The estimator shrinks regression coefficients of some of the variables to zero, hence selecting essential variables. The penalty parameter lambda determining feature selection was chosen by tenfold cross-validation to minimize predicted mean-squared error. Model averaging is a technique based on the empirical distribution of the statistics resulting from the resampling of the original population with a replacement. We used 2step model averaging. In the first step, the model selection using LASSO shrinkage was repeated for 1,000 samples. The procedure allowed for ranking of variable importance by reporting the percentage of time that a variable was selected into the model. This first step of model averaging produced a model that contained a large number of effects. The second step of the model averaging (ie, refitting) was used to obtain a more parsimonious model by specifying the percentage of cut-point of effects retained in the final model. After a more parsimonious model was identified, a least-squares model was fit with no effect selection on 1,000 samples with replacement, which produced an empirical distribution of the regression coefficients on which the importance of the variables was based. Because standard inference does not properly consider the model selection process in LASSO, model averaging is the preferred method to interpret the standard error of the model estimates (22). The list of essential factors for both outcomes is reported using $20 \%$ and $40 \%$ frequency selection. The lower percentage was less restrictive and allowed more variables into the model. Based on the empirical distribution, the mean value of regression coefficients with a $90 \%$ confidence interval was reported as the final model results. All statistical analyses were done by using SAS 9.4 (SAS Institute).

We also tested a full linear model, allowing all 32 variables to be present, which represented the least-biased parameter estimates. All models controlled for partnering site using a set of indicator variables. The caregiver, child, and household demographic characteristics and children's brushing behaviors are described elsewhere (19).

\section{Results}

The mean child age was 21.5 (SD 6.9) months, and $50.7 \%$ of children were female. Almost all children had health insurance (95.5\%), which was mainly Medicaid (89.3\%). Most caregivers were female (96.4\%) and the biological parent (96.4\%); the rest were other relatives or foster parents. Parents described themselves primarily as Black race $(41.9 \%)$ or Hispanic ethnicity $(52.1 \%)$. More than half reported some education after high school $(52.4 \%), 31.4 \%$ had a high school degree or GED, and $16.2 \%$ had less than a high school education. Sixty-one percent of caregivers lived with a partner or spouse. Caregivers reported their overall health as "excellent/very good" (40.5\%), "good" (39.8\%), or "fair/ poor" (19.8\%). Caregivers reported much worse social functioning (mean $t$-score, 32.0 [SD, 6.9]) than the reference population (mean $t$-score, 50 [SD, 10]). Caregiver anxiety and depression symptoms were slightly lower than the reference population means (mean $t$-score, 46.6 [SD, 8.1] and mean $t$-score, 46.2 [SD, 6.9], respectively).

Only $25(6.0 \%)$ caregivers had not started brushing or wiping their children's teeth. For the rest, $45.0 \%$ brushed their children's teeth twice per day or more, $33.8 \%$ once per day, and $15.2 \%$ brushed sometimes but not every day. The mean OHI-MIS plaque score was in the range of "poor" at 1.9 (SD 0.6); $54.9 \%$ scored 1.9 or

The opinions expressed by authors contributing to this journal do not necessarily reflect the opinions of the U.S. Department of Health and Human Services, the Public Health Service, the Centers for Disease Control and Prevention, or the authors' affiliated institutions. 
higher. Most caregivers did all the brushing during observations without active child participation (6.2\%). Most children (74.3\%) had a child-sized toothbrush, and 52.3\% used toothpaste with fluoride. Some children rinsed with water (36.0\%) and/or spit $(25.3 \%)$ after brushing.

Caregivers reported high oral health knowledge (mean, 4.2 [SD $0.8]$ ), and social support was comparable to reference population means (Table 1). Half of the caregivers reported that the activities of daily life never made it difficult to care for their child's teeth, although $26.0 \%$ of caregivers never or rarely had help caring for their children's teeth. More than half of children (59.7\%) had never been to the dentist. Exclusively drinking purchased bottled water was the most common response for drinking water source (54.6\%). Exposure to sugary beverages was common, with $28.8 \%$ saying their children consumed sugary beverages once per day and $37.2 \%$ consuming sugary beverages twice per day or more. Caregivers reported major oral health challenges of their own; $56.4 \%$ said their mouth and teeth were in "fair" or "poor" condition. One quarter $(25.7 \%)$ brushed less than twice per day, and $43.2 \%$ had not been to a dentist in over a year. The main reasons for caregivers not getting needed dental care in the past year were related to cost and insurance coverage.

Tables 2 and 3 present the results of multiple linear models without selection (full models) and the variables selected using LASSO regularization from the 32 potential associated factors, controlling for partner sites. The overlap in variables identified as important between the full models and LASSO regularization indicates low confounding and multicollinearity between variables. The LASSO $20 \%$ frequency selection shows the less restricted model, which allowed more variables to remain. The important factors for frequency of child tooth brushing identified by the more restrictive LASSO regularization with a $40 \%$ threshold to be retained in the final model included 8 variables, 7 of which met significance at the $10 \%$ level. Child brushing frequency (Table 2) was higher when children were older (mean $\beta=0.014 ; 90 \% \mathrm{CI}$, 0.006 to 0.022 ); used the correct toothpaste amount (mean $\beta=$ $0.115 ; 90 \% \mathrm{CI}, 0.004$ to 0.221 ); brushed for a longer duration (mean $\beta=0.001 ; 90 \% \mathrm{CI}, 0.000$ to 0.002 ); and when caregivers brushed their own teeth more frequently (mean $\beta=0.397 ; 90 \% \mathrm{CI}$, 0.299 to 0.490 ), had more help with the overall care of the child's teeth and child brushing (mean $\beta=0.058 ; 90 \% \mathrm{CI}, 0.021$ to 0.096), and had family or a partner to help care for the child's teeth (mean $\beta=0.292 ; 90 \%$ CI, 0.229 to 0.354 ). Child brushing frequency was lower for caregivers with more brushing interference from activities of daily life (mean $\beta=-0.105 ; 10 \% \mathrm{CI}$, -0.161 to -0.048$)$.
With regard to plaque score, children whose caregivers had more help from other adults with brushing their child's teeth had better plaque scores (mean $\beta=-0.092 ; 90 \% \mathrm{CI},-0.156$ to -0.028 ) (Table 3). Higher plaque scores were seen in children with higher sugary beverage consumption (mean $\beta=0.014 ; 10 \% \mathrm{CI}, 0.006$ to 0.022 ), higher sweet or sugary food consumption (mean $\beta=0.009$; $90 \% \mathrm{CI}, 0.001$ to 0.017 ), and lower household incomes (mean $\beta=$ $0.153 ; 90 \% \mathrm{CI}, 0.036$ to 0.268$)$.

\section{Discussion}

We identified multiple factors associated with tooth brushing behaviors and dental plaque in low-income children aged 36 months or younger, and these findings are relevant because dental caries begins early. The consistency in selecting the same set of factors between the full and LASSO regularization models highlights the robustness of the selection procedure in identifying meaningful factors associated with the frequency of tooth brushing and plaque score for this population.

Data from 2011-2016 reported a caries prevalence of $23 \%$ in US children aged 2 to 5 years, and this prevalence doubled by elementary school (1). In Illinois, overall caries and untreated caries prevalence have repeatedly surpassed national rates and disproportionately impact low-income, non-Hispanic Black, and Hispanic children (23). The participants in our analyses represent this highrisk demographic category. Identification of early risk and protective factors is essential to reduce oral health disparities and prevent or slow caries development in children.

The most influential factors associated with child brushing frequency in our analyses were the caregiver's own brushing frequency and caregivers having assistance with brushing from others. These associations are consistent with findings from other studies (24-26). Caregivers that brush their own teeth are more likely to brush their children's teeth as well (26). This association may be driven by caregiver oral health literacy, an overall value on oral hygiene within the family, established household routines, or by the fundamental principle that children learn from imitating adults (27). Having additional caregivers assist with child tooth brushing was associated with both higher brushing frequency and lower plaque scores. This points to the critical need for more family support for child brushing at this young age, mainly because children do not have the knowledge or manual dexterity to brush their teeth independently until they are much older. Caregivers have a fixed amount of time to complete necessary tasks, such as those conducted as part of morning or evening routines. When additional caregivers are available to assist with these tasks, children

\footnotetext{
The opinions expressed by authors contributing to this journal do not necessarily reflect the opinions of the U.S. Department of Health and Human Services, the Public Health Service, the Centers for Disease Control and Prevention, or the authors' affiliated institutions.
} 
are more likely to receive assistance or supervision with an oral health regimen. Our findings emphasize the importance of the family unit or household, as everyone plays an important role in encouraging and directly supervising a child's tooth brushing.

The frequency of consumption of sugary foods and beverages was associated with worse plaque scores. This finding may be because parents that give more sugary beverages may demonstrate other unhealthy behaviors such as brushing less frequently or effectively. Households that rely on calorie-dense, readily available foods may do so out of necessity and not have the capacity or support to implement regular brushing routines. This finding is concerning because the frequency of exposure to sugar-sweetened foods and beverages is a significant risk factor for dental caries via acidogenic bacteria in plaque (28).

Finally, children in the lowest income category had the highest levels of plaque. Although most of our sample was low-income, worse outcomes in the lowest income level are not surprising. Low-income caregivers have repeatedly reported significant barriers to accessing dental care for their children (29), and these results are compounded by the lack of providers that accept Medicaid, as well as limited case management resources. The overall rate of providers enrolled with Medicaid in the Chicago area is high compared with the rest of the state, mirroring the geographic density of Illinois's population. Unfortunately, enrollment as a Medicaid provider does not mean these dentists serve a significant number of patients on Medicaid. The reality is that many of these providers take only a small number of Medicaid patients and may not perform restorative procedures.

What was surprising were the many factors not associated with brushing behaviors, including access to dental care, caregiver quality of life, social functioning, and caregiver oral health knowledge. Research indicates that children's dental care usage behaviors were associated with their caregivers' behaviors in these areas; children were more likely to have used dental care within the past year when their parents also used dental services $(23,25)$. A possible explanation for the lack of association between dental care use and child brushing behaviors is that most of our sample had the same insurance, limiting variability. Research shows that the overall physical and psychological health and functioning of caregivers influences how they care for the health of their children; poor health, adversity, and inequality accumulate over the life course and across generations (30). Our study did not show differences in behaviors associated with caregiver quality of life and mental health, which may have been because of a lack of variability in the sample, instrument limitations, or perhaps not-yetidentified resilience factors. Finally, uncooperative child behavior is common in toddlers and poses a barrier to tooth brushing, even when caregiver knowledge and intent are good.
Our study has limitations. Because the data were cross-sectional, causation and potential directionality of effects cannot be established. We also did not measure all modifiable factors that influence oral health behaviors. The sample was limited to 1 densely populated urban county in the Midwest, and families had similar economic and races/ethnicities, which limits generalizability. Tooth brushing frequency was caregiver-reported because of the challenges of objectively measuring this behavior in young children, raising the potential for social desirability bias and data inaccuracy. However, we compared our self-reported data to data from other studies, including NHANES, and our results were similar (19). We also added a second measure of brushing — plaque score - to objectively capture the adequacy of brushing behaviors.

Our results indicate the necessity of interventions that target adult assistance with child brushing and reduction of sugary beverages and snack consumption among very young children. Similar to results in older children, our results demonstrate that brushing behaviors of young children are strongly associated with those of their parents and the level of family support for brushing. Interventions to improve brushing in young children should focus on the entire family, encouraging healthy oral health behaviors for parents as well as children. Clinicians and educators should also consider asking about family routines and supports parents have for brushing their children's teeth and offering appropriate interventions when problems are identified. Because low-income urban children are at high risk for developing caries beginning at a very early age, research is needed to determine whether these risk factors are also associated with caries development over time. We should also continue to develop and test interventions that will translate into improved oral health behaviors and outcomes for children.

\section{Acknowledgments}

This work was supported by the National Institute of Dental and Craniofacial Research of the National Institutes of Health (Grant No. UH3DE025483, Principal Investigator, Molly A. Martin.) We thank the other members of the CO-OP Chicago Steering Committee who did not participate as authors, including Michael Berbaum, Jennifer Bereckis, Marcio da Fonseca, William Frese, Mark Minier, Jennie Pinkwater, Sheela Raja, Anna Sandoval, and Rebecca Van Horn. A special thanks is offered to Gizelle Alvarez, Anabelen Diaz, Nadia Ochoa, Nia O'Neal, and Nusirat Williams, who collected the data, and our community health workers Melissa Hernandez Contreras, Monserrath Espinosa, Hope Opuada, and Mayra Pereddo. Our community advisory board (https://co-opchicago.ihrp.uic.edu/) provided support and guidance.

The opinions expressed by authors contributing to this journal do not necessarily reflect the opinions of the U.S. Department of Health and Human Services, the Public Health Service, the Centers for Disease Control and Prevention, or the authors' affiliated institutions. 
Finally, we thank the families, staff, providers, and administrators at our partner clinics and WIC centers: Aunt Martha's Pediatric Health and Wellness Center, Aunt Martha's South Holland Community Health Center, Aunt Martha's Southeast Side Community Health Center, Chicago Department of Public Health (CDPH) WIC Friend Family Health Center, CDPH WIC Greater Lawn Health Center, and CDPH WIC Westside Health Partnership, The Community and Economic Development Association of Cook County, Inc (CEDA) WIC Blue Island, CEDA WIC Diversey, CEDA WIC Harvey, CEDA WIC Irving Park, CEDA WIC Maywood, CEDA WIC Oak Park, CEDA WIC Summit, Mile Square Health Center Back of the Yards, Mile Square Health Center Cicero, Mile Square Health Center Englewood, Mile Square Health Center Main, Mile Square Health Center South Shore, UI Health Child and Youth Center, and Vida Pediatrics.

\section{Author Information}

Corresponding Author: Molly A Martin, MD, MAPP, University of Illinois at Chicago, 840 South Wood St, M/C 856, Chicago, IL 60612. Telephone: 312-996-2363. Email: mollyma@uic.edu.

Author Affiliations: ${ }^{1}$ College of Medicine, Department of Pediatrics, University of Illinois at Chicago, Chicago, Illinois. ${ }^{2}$ Institute for Health Research and Policy, University of Illinois at Chicago, Chicago, Illinois. ${ }^{3}$ College of Dentistry, Department of Pediatrics, University of Illinois at Chicago, Chicago, Illinois. ${ }^{4}$ College of Medicine, Department of Anesthesiology, University of Illinois at Chicago, Chicago, Illinois.

\section{References}

1. Centers for Disease Control and Prevention. Oral health surveillance report: trends in dental caries and sealants, tooth retention, and edentulism, United States, 1999-2004 to 2011-2016. Atlanta (GA): US Dept of Health and Human Services, Centers for Disease Control and Prevention; 2019.

2. Guarnizo-Herreño CC, Lyu W, Wehby GL. Children's oral health and academic performance: evidence of a persisting relationship over the last decade in the United States. J Pediatr 2019;209:183-189.e2.

3. Casamassimo PS, Thikkurissy S, Edelstein BL, Maiorini E. Beyond the dmft: the human and economic cost of early childhood caries. J Am Dent Assoc 2009;140(6):650-7.

4. Sheiham A. Dental caries affects body weight, growth and quality of life in pre-school children. Br Dent J 2006; 201(10):625-6.
5. Schwendicke F, Dörfer CE, Schlattmann P, Foster Page L, Thomson WM, Paris S. Socioeconomic inequality and caries: a systematic review and meta-analysis. J Dent Res 2015; 94(1):10-8.

6. Mouradian WE, Wehr E, Crall JJ. Disparities in children's oral health and access to dental care. JAMA 2000 ; 284(20):2625-31.

7. Shariff JA, Edelstein BL. Medicaid meets its equal access requirement for dental care, but oral health disparities remain. Health Aff (Millwood) 2016;35(12):2259-67.

8. Fisher-Owens SA, Isong IA, Soobader MJ, Gansky SA, Weintraub JA, Platt LJ, et al. An examination of racial/ethnic disparities in children's oral health in the United States. J Public Health Dent 2013;73(2):166-74.

9. Crall JJ, Forest CB. A Life Course Health Development Perspective on Oral Health. New York (NY): Springer Publishing; 2018.

10. Broadbent JM, Zeng J, Foster Page LA, Baker SR, Ramrakha $\mathrm{S}$, Thomson WM. Oral health-related beliefs, behaviors, and outcomes through the life course. J Dent Res 2016 ; 95(7):808-13.

11. Section On Oral Health. Maintaining and improving the oral health of young children. Pediatrics 2014;134(6):1224-9.

12. Fisher-Owens SA, Gansky SA, Platt LJ, Weintraub JA, Soobader MJ, Bramlett MD, et al. Influences on children's oral health: a conceptual model. Pediatrics 2007;120(3):e510-20.

13. Fisher-Owens SA, Mertz E. Preventing oral disease: alternative providers and places to address this commonplace condition. Pediatr Clin North Am 2018;65(5):1063-72.

14. Bader JD, Rozier RG, Lohr KN, Frame PS. Physicians' roles in preventing dental caries in preschool children: a summary of the evidence for the U.S. Preventive Services Task Force. Am J Prev Med 2004;26(4):315-25.

15. American Dental Association Council on Scientific Affairs. Fluoride toothpaste use for young children. J Am Dent Assoc 2014;145(2):190-1.

16. Pahel BT, Rozier RG, Stearns SC, Quiñonez RB. Effectiveness of preventive dental treatments by physicians for young Medicaid enrollees. Pediatrics 2011;127(3):e682-9.

17. Kobayashi M, Chi D, Coldwell SE, Domoto P, Milgrom P. The effectiveness and estimated costs of the access to baby and child dentistry program in Washington State. J Am Dent Assoc 2005;136(9):1257-63.

18. Eklund SA, Pittman JL, Clark SJ. Michigan Medicaid's Healthy Kids Dental program: an assessment of the first 12 months. J Am Dent Assoc 2003;134(11):1509-15.

The opinions expressed by authors contributing to this journal do not necessarily reflect the opinions of the U.S. Department of Health and Human Services, the Public Health Service, the Centers for Disease Control and Prevention, or the authors' affiliated institutions. 
19. Martin MA, Zimmerman LJ, Rosales GF, Lee HH, Songthangtham N, Pugach O, et al. Design and sample characteristics of COordinated Oral health Promotion (CO-OP) Chicago: A cluster-randomized controlled trial. Contemp Clin Trials 2020;92:105919.

20. Greene JC, Vermillion JR. The Simplified Oral Hygiene Index. J Am Dent Assoc 1964;68(1):7-13.

21. Wei SH, Lang NP. Periodontal epidemiological indices for children and adolescents: II. Evaluation of oral hygiene; III. Clinical applications. Pediatr Dent 1982;4(1):64-73.

22. Efron B. Estimation and accuracy after model selection. J Am Stat Assoc 2014;109(507):991-1007.

23. Illinois Department of Public Health. Healthy Smiles Healthy Growth 2018-19: an assessment of oral health status, beverage consumption and body mass index of third-grade children in Illinois. 2020. http://www.dph.illinois.gov/sites/default/files/ publications/hshg-201819-report-final-2-21-2020.pdf. Accessed May 5, 2020.

24. Isong IA, Zuckerman KE, Rao SR, Kuhlthau KA, Winickoff JP, Perrin JMI. Association between parents' and children's use of oral health services. Pediatrics 2010;125(3):502-8.

25. Darmawikarta D, Chen Y, Carsley S, Birken CS, Parkin PC, Schroth RJ, et al.; TARGet Kids! Collaboration. Factors associated with dental care utilization in early childhood. Pediatrics 2014;133(6):e1594-600.

26. Wilson AR, Brega AG, Campagna EJ, Braun PA, Henderson WG, Bryant LL, et al. Validation and impact of caregivers' oral health knowledge and behavior on children's oral health status. Pediatr Dent 2016;38(1):47-54.

27. Miller E, Lee JY, DeWalt DA, Vann WF Jr. Impact of caregiver literacy on children's oral health outcomes. Pediatrics 2010;126(1):107-14.

28. Marshall TA. Preventing dental caries associated with sugarsweetened beverages. J Am Dent Assoc 2013; 144(10):1148-52.

29. Isong IA, Luff D, Perrin JM, Winickoff JP, Ng MW. Parental perspectives of early childhood caries. Clin Pediatr (Phila) 2012;51(1):77-85.

30. Wickrama KA, Conger RD, Abraham WT. Early adversity and later health: the intergenerational transmission of adversity through mental disorder and physical illness. J Gerontol B Psychol Sci Soc Sci 2005;60(Speciallssue 2):125-9.

31. Albino J, Tiwari T, Gansky SA, Henshaw MM, Barker JC, Brega AG, et al. The basic research factors questionnaire for studying early childhood caries. Early Childhood Caries Collab Cent 2017;17(1):83.

The opinions expressed by authors contributing to this journal do not necessarily reflect the opinions of the U.S. Department of Health and Human Services, the Public Health Service, the Centers for Disease Control and Prevention, or the authors' affiliated institutions. 


\section{Tables}

Table 1. Oral Health Risk Factors of Children Aged 6 to 36 Months $(\mathrm{N}=420),{ }^{a}$ Coordinated Oral Health Promotion Chicago Study, Chicago, Illinois, $2018-2019$

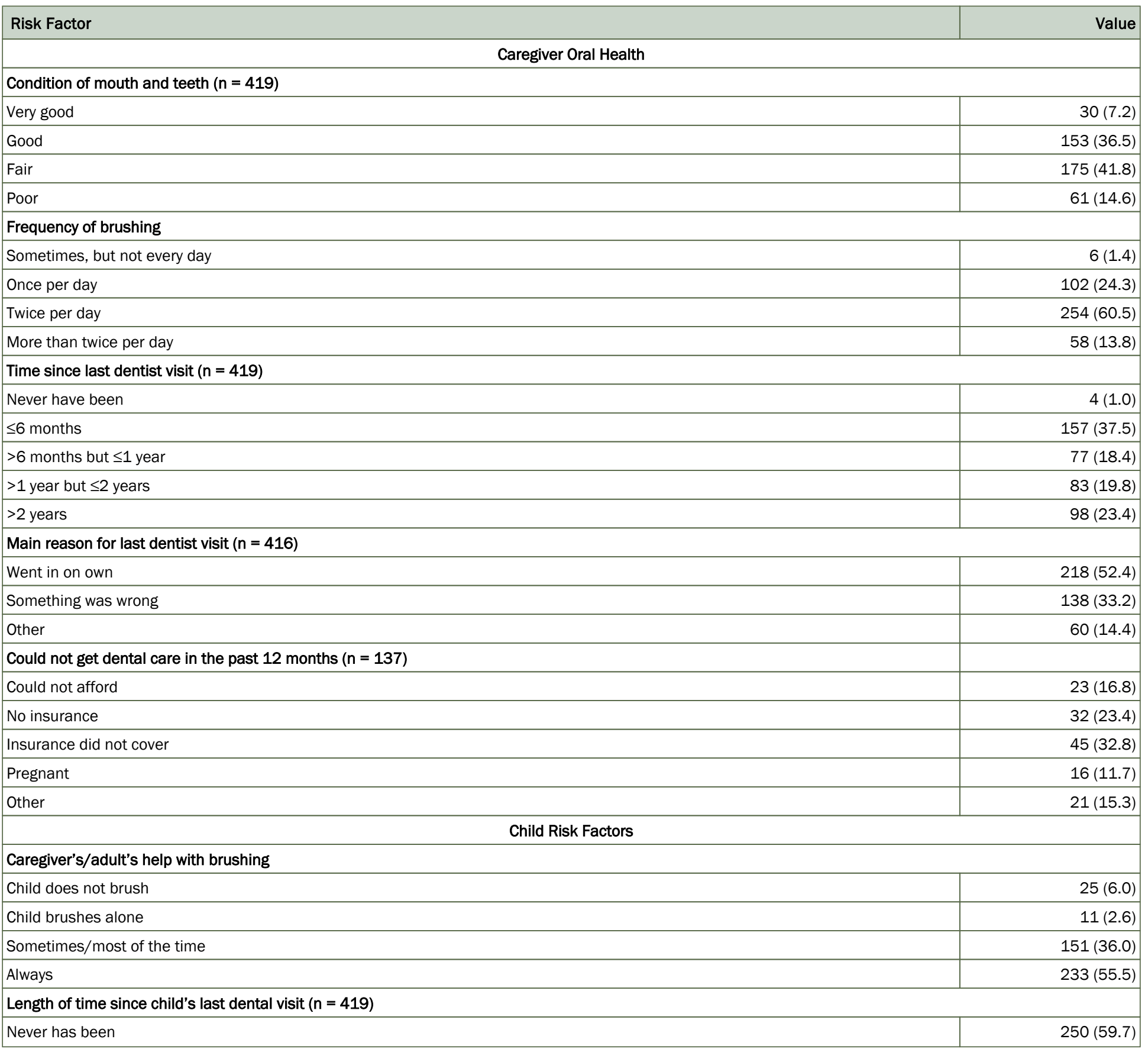

${ }^{a}$ Values are no. (\%), unless otherwise indicated; $\mathrm{N}=420$ unless otherwise indicated.

${ }^{\mathrm{b}}$ The Oral Health Knowledge Scale was developed by the Knowledge and Behavior Workgroup of the Early Childhood Caries Collaborating Centers (31).

\footnotetext{
The opinions expressed by authors contributing to this journal do not necessarily reflect the opinions of the U.S. Department of Health and Human Services, the Public Health Service, the Centers for Disease Control and Prevention, or the authors' affiliated institutions.
} 
(continued)

Table 1. Oral Health Risk Factors of Children Aged 6 to 36 Months $(\mathrm{N}=420),{ }^{a}$ Coordinated Oral Health Promotion Chicago Study, Chicago, Illinois, $2018-2019$

\begin{tabular}{|c|c|}
\hline Risk Factor & Value \\
\hline$\leq 6$ months & $139(33.2)$ \\
\hline$>6$ months but $\leq 2$ years & $30(7.2)$ \\
\hline Child needed dental care but could not get, past 12 months & $31(7.4)$ \\
\hline \multicolumn{2}{|l|}{ Type of drinking water $(n=416)$} \\
\hline Purchased only & $227(54.6)$ \\
\hline Tap only & $73(17.5)$ \\
\hline Both purchased and tap & $116(27.9)$ \\
\hline \multicolumn{2}{|l|}{ Frequency of sugary beverage consumption } \\
\hline Rarely or never & $74(17.6)$ \\
\hline Once per week, not daily & $69(16.4)$ \\
\hline Once per day & $121(28.8)$ \\
\hline Twice per day & $83(19.8)$ \\
\hline Three times per day or more & $73(17.4)$ \\
\hline Child 15 months or older and still drinks from bottle $(n=341)$ & $137(40.2)$ \\
\hline \multicolumn{2}{|c|}{ Caregiver Knowledge, Support, and Barriers } \\
\hline Caregiver knowledge, mean (SD) ${ }^{b}$ & $4.2(0.8)$ \\
\hline \multicolumn{2}{|l|}{ Family/partner help care for child's teeth } \\
\hline All the time & $144(34.3)$ \\
\hline Most of the time & $83(19.8)$ \\
\hline Some of the time & $84(20.0)$ \\
\hline Rarely & $42(10.0)$ \\
\hline Never & $67(16.0)$ \\
\hline \multicolumn{2}{|l|}{ Social support, $t$-score, mean (SD) $(n=419)$} \\
\hline Emotional & $55.9(8.9)$ \\
\hline Instrumental & $54.8(9.3)$ \\
\hline Informational & $57.7(9.8)$ \\
\hline \multicolumn{2}{|l|}{ Activities of daily life make it difficult to care for child's teeth } \\
\hline All the time & $7(1.7)$ \\
\hline Most of the time & $29(6.9)$ \\
\hline Some of the time & $84(20.0)$ \\
\hline Rarely & $88(21.0)$ \\
\hline Never & $212(50.5)$ \\
\hline
\end{tabular}

${ }^{\text {a }}$ Values are no. (\%), unless otherwise indicated; $N=420$ unless otherwise indicated.

${ }^{b}$ The Oral Health Knowledge Scale was developed by the Knowledge and Behavior Workgroup of the Early Childhood Caries Collaborating Centers (31). 
Table 2. Factors Associated with Frequency of Child Tooth Brushing Among Children Aged 6 to 36 Months ( $=420$ ), Coordinated Oral Health Promotion Chicago Study, Chicago, Illinois, 2018-2019

\begin{tabular}{|c|c|c|c|c|c|c|c|c|c|}
\hline \multirow[b]{2}{*}{ Variable } & \multicolumn{3}{|c|}{ Full Model } & \multicolumn{3}{|c|}{ LASSO $20 \%$ Frequency Selection } & \multicolumn{3}{|c|}{ LASSO $40 \%$ Frequency Selection } \\
\hline & $\beta$ & SD & $10 \% \mathrm{Cl}$ & Mean $\beta$ & SD & $90 \% \mathrm{Cl}$ & Mean $\beta$ & SD & $90 \% \mathrm{Cl}$ \\
\hline Intercept & 0.423 & 0.649 & -0.644 to 1.489 & $-0.610^{b}$ & 0.253 & -1.02 to -0.164 & $-0.613^{b}$ & 0.139 & -0.835 to -0.381 \\
\hline $\begin{array}{l}\text { Activities of daily life make it difficult to } \\
\text { care for child's teeth }\end{array}$ & $-0.127^{b}$ & 0.033 & -0.182 to -0.072 & $-0.102^{b}$ & 0.035 & -0.158 to -0.043 & $-0.105^{b}$ & 0.034 & -0.161 to -0.048 \\
\hline Caregiver age in years & 0.002 & 0.006 & -0.007 to 0.012 & 0.003 & 0.005 & -0.005 to 0.012 & - & - & - \\
\hline Caregiver/adults help with brushing & $0.282^{b}$ & 0.041 & 0.214 to 0.349 & $0.273^{b}$ & 0.040 & 0.206 to 0.339 & $0.292^{b}$ & 0.037 & 0.229 to 0.354 \\
\hline Caregiver frequency of brushing & $0.378^{b}$ & 0.055 & 0.287 to 0.468 & $0.386^{b}$ & 0.055 & 0.294 to 0.474 & $0.397^{b}$ & 0.058 & 0.299 to 0.490 \\
\hline Child age in months & $0.015^{\mathrm{b}}$ & 0.006 & 0.006 to 0.025 & $0.014^{b}$ & 0.006 & 0.005 to 0.023 & $0.014^{b}$ & 0.005 & 0.006 to 0.022 \\
\hline Correct toothpaste amount & 0.098 & 0.071 & -0.019 to 0.214 & 0.082 & 0.072 & -0.031 to 0.206 & $0.115^{b}$ & 0.068 & 0.004 to 0.221 \\
\hline Family/partner help care for child's teeth & $0.078^{b}$ & 0.025 & 0.037 to 0.118 & $0.058^{b}$ & 0.023 & 0.019 to 0.096 & $0.058^{b}$ & 0.023 & 0.021 to 0.096 \\
\hline Fluoride toothpaste used & 0.004 & 0.071 & -0.113 to 0.122 & 0.032 & 0.068 & -0.082 to 0.145 & - & - & - \\
\hline Frequency of sweet or sugary foods & -0.004 & 0.005 & -0.012 to 0.005 & -0.005 & 0.004 & -0.012 to 0.003 & - & - & - \\
\hline Household chaos & -0.089 & 0.064 & -0.194 to 0.016 & -0.042 & 0.062 & -0.147 to 0.059 & - & - & - \\
\hline \multicolumn{10}{|l|}{ Household income in last year, $\$$} \\
\hline$<30,000$ & 0.091 & 0.081 & -0.042 to 0.223 & 0.107 & 0.080 & -0.026 to 0.238 & 0.100 & 0.078 & -0.029 to 0.236 \\
\hline $30,000-60,000$ & -0.057 & 0.093 & -0.210 to 0.095 & - & - & - & - & - & - \\
\hline$>60,000$ & -0.144 & 0.144 & -0.381 to 0.093 & - & - & - & - & - & - \\
\hline Unknown/refused & & & 1 [Reference] & - & - & - & - & - & - \\
\hline Length of time since child's last dental visit & 0.065 & 0.056 & -0.028 to 0.158 & 0.047 & 0.054 & -0.043 to 0.136 & - & - & - \\
\hline Observed brushing time in seconds & $0.001^{b}$ & 0.001 & 0.000 to 0.002 & $0.001^{b}$ & 0.001 & 0.000 to 0.002 & $0.001^{b}$ & 0.001 & 0.000 to 0.002 \\
\hline Total ECOHIS Score & 0.008 & 0.008 & -0.005 to 0.021 & $0.012^{b}$ & 0.007 & 0.000 to 0.024 & - & - & - \\
\hline
\end{tabular}

Abbreviations: -, not applicable; ECOHIS, Early Childhood Oral Health Impact Scale; LASSO, Least Absolute Shrinkage and Selection Operator.

${ }^{a}$ Models include 32 variables; only significant variables are reported in the table. All models also control for a partner site. The full model uses categorical variables as a single construct, whereas LASSO treats the set of indicator variables from the same categorical variables as independent variables. The coefficients for the household income variable represent differences from the reference category in the full model, but in the LASSO models the coefficients represent differences from all categories not selected into the model.

${ }^{\mathrm{b}}$ Significant at $P<.10$. 
Table 3. Factors Associated with Higher Child Plaque Score Among Children Aged 6 to 36 Months $(\mathrm{N}=420)$, Coordinated Oral Health Promotion Chicago Study, Chicago, Illinois, 2018-2019

\begin{tabular}{|c|c|c|c|c|c|c|c|c|c|}
\hline \multirow[b]{2}{*}{ Factor } & \multicolumn{3}{|c|}{ Full Model } & \multicolumn{3}{|c|}{ LASSO 20\% Frequency Selection } & \multicolumn{3}{|c|}{ LASSO $40 \%$ Frequency Selection } \\
\hline & $\beta$ & SD & $10 \% \mathrm{Cl}$ & Mean $\beta$ & SD & $90 \% \mathrm{Cl}$ & Mean $\beta$ & SD & $90 \% \mathrm{Cl}$ \\
\hline Intercept & $1.717^{\mathrm{b}}$ & 0.628 & 0.684 to 2.749 & $1.939^{b}$ & 0.204 & 1.593 to 2.276 & $2.010^{b}$ & 0.107 & 1.833 to 2.181 \\
\hline Caregiver/adults help with brushing & $-0.094^{b}$ & 0.040 & -0.159 to -0.028 & $-0.102^{b}$ & 0.040 & -0.167 to -0.034 & $-0.092^{b}$ & 0.040 & -0.156 to -0.028 \\
\hline Caregiver age in years & -0.005 & 0.005 & -0.013 to 0.004 & -0.004 & 0.005 & -0.012 to 0.004 & - & - & - \\
\hline \multicolumn{10}{|l|}{ Caregiver relationship status } \\
\hline Single & 0.261 & 0.147 & 0.019 to 0.503 & 0.114 & 0.071 & -0.007 to 0.225 & \multirow{2}{*}{-} & \multirow{2}{*}{-} & - \\
\hline Living with partner/spouse & 0.134 & 0.140 & -0.096 to 0.365 & - & - & - & & & - \\
\hline Separated/divorced & & & 1 [Reference] & - & - & - & - & - & - \\
\hline \multicolumn{10}{|l|}{ Child race/ethnicity } \\
\hline Black & 0.066 & 0.155 & -0.189 to 0.322 & - & - & - & \multirow{3}{*}{-} & \multirow{3}{*}{-} & - \\
\hline Hispanic & 0.198 & 0.154 & -0.056 to 0.450 & $0.127^{b}$ & 0.073 & 0.008 to 0.252 & & & - \\
\hline Other & 0.372 & 0.254 & -0.046 to 0.791 & $0.347^{b}$ & 0.181 & 0.040 to 0.628 & & & - \\
\hline White & \multicolumn{3}{|r|}{1 [Reference] } & - & - & - & - & - & - \\
\hline Observed brushing time in seconds & $-0.001^{b}$ & 0.001 & -0.002 to -0.000 & $-0.001^{b}$ & 0.001 & -0.002 to -0.000 & -0.001 & 0.001 & -0.002 to 0.000 \\
\hline Fluoride toothpaste used & 0.093 & 0.069 & -0.021 to 0.207 & $0.109^{b}$ & 0.065 & 0.002 to 0.218 & - & - & - \\
\hline Frequency of sugary beverage consumption & $0.014^{b}$ & 0.005 & 0.006 to 0.023 & $0.014^{b}$ & 0.005 & 0.005 to 0.022 & $0.014^{b}$ & 0.005 & 0.006 to 0.022 \\
\hline Frequency of sweet/sugary foods & 0.008 & 0.005 & -0.000 to 0.016 & 0.007 & 0.005 & -0.001 to 0.015 & $0.009^{b}$ & 0.005 & 0.001 to 0.017 \\
\hline \multicolumn{10}{|l|}{ Household income in last year, $\$$} \\
\hline$<30,000$ & $0.187^{b}$ & 0.078 & 0.058 to 0.315 & $0.175^{b}$ & 0.074 & 0.049 to 0.292 & $0.153^{b}$ & 0.071 & 0.036 to 0.268 \\
\hline $30,000-60,000$ & -0.004 & 0.090 & -0.152 to 0.144 & - & - & - & - & - & - \\
\hline$>60,000$ & 0.007 & 0.139 & -0.222 to 0.236 & - & - & - & - & - & - \\
\hline Unknown/refused & \multicolumn{3}{|r|}{1 [Reference] } & - & - & - & - & - & - \\
\hline Total ECOHIS Score & 0.007 & 0.007 & -0.005 to 0.019 & 0.005 & 0.007 & -0.006 to 0.017 & - & - & - \\
\hline
\end{tabular}

Abbreviations: -, not applicable; ECOHIS, Early Childhood Oral Health Impact Scale; LASSO, Least Absolute Shrinkage and Selection Operator.

${ }^{a}$ Models include 32 factors; only significant variables are reported in the table. All models also control for a partner site. The full model uses categorical variables as a single construct, whereas LASSO treats the set of indicator variables from the same categorical variables as independent variables. In this model, for caregiver race/ethnicity, caregiver relationship status, and household income, the coefficients in the full model represent differences from the reference category but in the LASSO models, the coefficients represent differences from all categories not selected into the model.

${ }^{\mathrm{b}}$ Significant at $P<.10$. 